\title{
Some fixed point theorems in 2-Banach spaces and 2-normed tensor product spaces
}

\author{
Dipankar Das ${ }^{1}$, Nilakshi Goswami ${ }^{1}$, Vandana ${ }^{2}$ \\ ${ }^{1}$ Department of Mathematics, Gauhati University, Guwahati, Assam, India \\ ${ }^{2}$ School of Studies in Mathematics, Pt. Ravishankar Shukla University, Raipur (C.G.), India
}

Received: 9 May 2016, Accepted: 28 July 2016

Published online: 5 January 2017.

\begin{abstract}
In this paper, we derive some fixed point theorems in 2-Banach spaces. Let $X$ be a 2-Banach space and $T$ be a self-mapping on $X$. Let $\psi:[0, \infty) \rightarrow[0, \infty) ; \beta, \phi:[0, \infty) \times[0, \infty) \rightarrow[0, \infty)$ and $\gamma:[0, \infty) \times[0, \infty) \times[0, \infty) \rightarrow[0, \infty)$ be continuous mappings having some specific characteristics. Using these mappings, we define some conditions for $T$ under which $T$ has a unique fixed point in $X$. The conditions for two self-mappings $T_{1}$ and $T_{2}$ on $X$ for having the common unique fixed point are also derived here with proper examples. Moreover, defining a 2-norm in the projective tensor product space, we derive a fixed point theorem here with a suitable example.
\end{abstract}

Keywords: 2-Banach space, fixed points, projective tensor product.

\section{Introduction}

In this paper, we derive some fixed point theorems for mappings on 2-Banach spaces satisfying some specific characteristics. The notion of 2-normed linear spaces and their topological structures was initiated by Gähler [10] in his paper "Linear 2-normed spaces". He studied the special class of 2-metric spaces which is linear and defined a 2-norm on those spaces. Motivated by this work, several authors namely Iseki [11], Rhoads [27], White [29], etc., studied various aspects of the fixed point theory and proved some fixed point theorems in 2-metric and 2-Banach spaces. Cho et al. [3] investigated about common fixed points of weakly compatible mappings in 2-metric spaces. In 1993, Khan and Khan [12] derived some results on fixed points of involution maps in 2-Banach spaces. In 2013 [28], Saha et al. discussed some fixed point theorems for a class of for weakly C-contractive mappings in a setting of 2-Banach Space.

\section{Preliminaries}

Definition 1. Let $X$ be a real linear space of dimension greater than 1 and let $\|.,$.$\| be a real valued function on X \times X$ satisfying the following conditions:

(i) $\|x, y\|=0$ if and only if $x$ and $y$ are linearly dependent,

(ii) $\|x, y\|=\|y, x\|$ for all $x, y \in X$,

(iii) $\|\alpha x, y\|=|\alpha|\|y, x\|$, $\alpha$ being real, $x, y \in X$,

(iv) $\|x, y+z\| \leqslant\|x, y\|+\|x, z\|$, for all, $x, y, z \in X$

Then $\|.,$.$\| is called a 2-norm on X$ and $(X,\|.,\|$.$) is called a linear 2-normed space.$

Definition 2. A sequence $\left\{x_{n}\right\}$ in a 2-normed space $(X,\|.,\|$.$) is said to be a Cauchy sequence if \lim _{n, m \rightarrow \infty}\left\|x_{n}-x_{m}, a\right\|=0$ for all $a$ in $X$. 
Definition 3. A sequence $\left\{x_{n}\right\}$ in a 2-normed space $X$ is called a convergent sequence if there is an $x$ in $X$ such that $\lim _{n \rightarrow \infty}\left\|x_{n}-x, a\right\|=0$ for all $a$ in $X$.

Definition 4. A 2-normed space in which every Cauchy sequence is convergent is called a 2-Banach space.

Definition 5. Let $X$ and $Y$ be two linear 2-normed spaces. An operator $T: X \rightarrow Y$ is said to be continuous at $x \in X$ if for every sequence $\left\{x_{n}\right\}$ in $X,\left\{x_{n}\right\} \rightarrow x$ as $n \rightarrow \infty$ implies $\left\{T\left(x_{n}\right)\right\} \rightarrow T(x)$ in $Y$ as $n \rightarrow \infty$.

Definition 6. Let $f$ and $g$ be two self-maps on a set $X$. If $f x=g x$, for some $x$ in $X$ then $x$ is called coincidence point of $f$ and $g$.

Definition 7. Let $f$ and $g$ be two self-maps defined on a set $X$. Then $f$ and $g$ are said to be weakly compatible if they commute at coincidence points, i.e., if $f u=g u$ for some $u \in X$, then $f g u=g f u$.

\section{Fixed point in 2-Banach spaces}

Theorem 1. Let $X$ be a 2-Banach space and $T$ be a self map on $X$. Let $\psi:[0, \infty) \rightarrow[0, \infty)$ and $\beta:[0, \infty) \times[0, \infty) \rightarrow[0, \infty)$ are continuous mappings satisfying the conditions: $\psi(0)=0, \psi$ is monotonically increasing;

$$
b \psi(s) \leqslant \beta(r, s) \Rightarrow b s \leqslant r, b \in\{1,2\} ; \beta(s, t)=0 \Leftrightarrow s=t=0 .
$$

Let

$$
\psi(\|T x-T y, a\|) \leqslant \beta(\|x-T x, a\|,\|y-T y, a\|)-\max [\psi(\|x-T x, a\|), \psi(\|y-T y, a\|)]
$$

where $x, y, a \in X$. Then $T$ has a unique fixed point on $X$.

Proof. For any fixed $x_{0} \in X$, we construct a sequence $\left\{x_{n}\right\}$ by $x_{n+1}=T x_{n}, n=0,1,2, \ldots$

$$
\begin{aligned}
\psi\left(\left\|x_{n}-x_{n+1}, a\right\|\right) & \leqslant \beta\left(\left\|x_{n-1}-x_{n}, a\right\|,\left\|x_{n}-x_{n+1}, a\right\|\right) \\
& -\max \left[\psi\left(\left\|x_{n-1}-x_{n}, a\right\|\right), \psi\left(\left\|x_{n}-x_{n+1}, a\right\|\right)\right] \\
& \leqslant \beta\left(\left\|x_{n-1}-x_{n}, a\right\|,\left\|x_{n}-x_{n+1}, a\right\|\right)
\end{aligned}
$$

Therefore we write,

$$
\left\|x_{n}-x_{n+1}, a\right\| \leqslant\left\|x_{n-1}-x_{n}, a\right\| .
$$

So, $\left\{\left\|x_{n}-x_{n+1}, a\right\|\right\}$ is a monotonic decreasing sequence of real numbers and hence it converges to some $r$, say, i.e., $\left\|x_{n}-x_{n+1}, a\right\| \rightarrow r$ as $n \rightarrow \infty$.

Now, $\left\|x_{n}-x_{n+1}, a\right\|=\left\|T x_{n-1}-T x_{n}, a\right\|$. So,

$$
\begin{aligned}
\psi(r) & =\psi\left(\lim _{n \rightarrow \infty}\left\|x_{n}-x_{n+1}, a\right\|\right)=\lim _{n \rightarrow \infty} \psi\left(\left\|T x_{n-1}-T x_{n}, a\right\|\right) \\
& \leqslant \lim _{n \rightarrow \infty}\left[\beta\left(\left\|x_{n-1}-T x_{n-1}, a\right\|,\left\|x_{n}-T x_{n}, a\right\|\right)-\max \left(\psi\left(\left\|x_{n-1}-T x_{n-1}, a\right\|\right), \psi\left(\left\|x_{n}-T x_{n}, a\right\|\right)\right)\right] \\
& =\lim _{n \rightarrow \infty}\left[\beta\left(\left\|x_{n-1}-x_{n}, a\right\|,\left\|x_{n}-x_{n+1}, a\right\|\right)-\max \left(\psi\left(\left\|x_{n-1}-x_{n}, a\right\|\right), \psi\left(\left\|x_{n}-x_{n+1}, a\right\|\right)\right)\right] \\
& =\beta(r, r)-\max [\psi(r), \psi(r)] .
\end{aligned}
$$

Thus, $2 \psi(r) \leqslant \beta(r, r) \Rightarrow 2 r \leqslant r$, possible for $r=0$. Hence, $\left\|x_{n}-x_{n+1}, a\right\| \rightarrow 0$ as $n \rightarrow \infty$.

Next, we show that $\left\{x_{n}\right\}$ is a Cauchy sequence in $X$. If possible, let $\left\{x_{n}\right\}$ be not a Cauchy sequence, and so, there exists 
$\varepsilon>0$ such that there exists sub sequences $\left\{x_{m_{k}}\right\}$ and $\left\{x_{n_{k}}\right\}$ of $\left\{x_{n}\right\}$ with $n_{k}>m_{k}>k$ such that $\left\|x_{m_{k}}-x_{n_{k}}, a\right\| \geqslant \varepsilon$ and $\left\|x_{m_{k}}-x_{n_{k}-1}, a\right\|<\varepsilon$ Then,

$$
\begin{aligned}
& \left.\psi(\varepsilon) \leqslant \psi\left\|x_{m_{k}}-x_{n_{k}}, a\right\|\right)=\psi\left(\left\|T x_{m_{k}-1}-T x_{n_{k}-1}, a\right\|\right) \\
& \leqslant \beta\left(\left\|x_{m_{k}-1}-T x_{m_{k}-1}, a\right\|,\left\|x_{n_{k}-1}-T x_{n_{k}-1}, a\right\|\right) \\
& \quad-\max \left[\psi\left(\left\|x_{m_{k}-1}-T x_{m_{k}-1}, a\right\|\right), \psi\left(\left\|x_{n_{k}-1}-T x_{n_{k}-1}, a\right\|\right)\right]
\end{aligned}
$$

Taking $n_{k}$ and $m_{k} \rightarrow \infty$ and using the continuity of $\beta$ and $\psi$

$$
\psi(\varepsilon) \leqslant \beta(0,0)-\max [\psi(0), \psi(0)]=0=\psi(0) \Rightarrow \varepsilon \leqslant 0
$$

which is a contradiction. Hence $\left\{x_{n}\right\}$ is a Cauchy sequence in $X$, and so, it converges to some $z$, in $X$. Now,

$$
\begin{aligned}
& \psi\left(\left\|x_{n}-T z, a\right\|\right) \leqslant \beta\left(\left\|x_{n-1}-T x_{n-1}, a\right\|,\|z-T z, a\|\right)-\max \left[\psi\left(\left\|x_{n-1}-T x_{n-1}, a\right\|\right), \psi(\|z-T z, a\|)\right] \\
& \Rightarrow 2 \psi(\|z-T z, a\|) \leqslant \beta(0,\|z-T z, a\|) ;[\operatorname{taking} n \rightarrow \infty] \\
& \Rightarrow 2\|z-T z, a\| \leqslant 0,(\forall a \in X) \\
& \Rightarrow\|z-T z, a\|=0
\end{aligned}
$$

Since $a$ is arbitrary, taking $a=0$, we get, $z=T z$.

To show the uniqueness: Let $T z_{1}=z_{1}$ and $T z_{2}=z_{2}$. Then

$$
\begin{aligned}
\psi\left(\left\|T z_{1}-T z_{2}, a\right\|\right) & \leqslant \beta\left(\left\|z_{1}-T z_{1}, a\right\|,\left\|z_{2}-T z_{2}, a\right\|\right)-\max \left[\psi\left(\left\|z_{1}-T z_{1}, a\right\|\right), \psi\left(\left\|z_{2}-T z_{2}, a\right\|\right)\right] \\
& =\beta(0,0)-\max [\psi(0), \psi(0)] .
\end{aligned}
$$

Therefore we write,

$$
\left\|T z_{1}-T z_{2}, a\right\|=0 \forall a \in X \Rightarrow T z_{1}=T z_{2} \Rightarrow z_{1}=z_{2} .
$$

The proof is completed.

Example 1. Let $X=\mathbb{R}^{3}$ and we consider the following 2-norm on $X$ (refer to [1])

$$
\|x, y\|=\left|\operatorname{det}\left(\begin{array}{ccc}
i & j & k \\
x_{1} & x_{2} & x_{3} \\
y_{1} & y_{2} & y_{3}
\end{array}\right)\right|
$$

where $x=\left(x_{1}, x_{2}, x_{3}\right), y=\left(y_{1}, y_{2}, y_{3}\right) \in \mathbb{R}^{3}$. Then $(X,\|.,\|$.$) is a 2-Banach space.$

We fix $(e, f, g) \in \mathbb{R}^{3}$ and let $T$ be a self mapping on $\mathbb{R}^{3}$ defined by $T(x, y, z)=(e, f, g) \forall(x, y, z) \in \mathbb{R}^{3}$.

Let $\psi(s)=2 s, \beta(r, s)=\frac{r}{2}+s ;$ where $(r, s) \in[0, \infty)$. Now, $T x=(e, f, g)=T y$ therefore $\|T x-T y, a\|=0$.

Hence all the conditions of Theorem 1 are satisfied. So, $T$ has a unique fixed point $(e, f, g) \in \mathbb{R}^{3}$.

For common fixed point of two self maps $T_{1}$ and $T_{2}$ on $X$, we prove. 
Theorem 2. Let $X$ be a 2-Banach space and $T_{1}$ and $T_{2}$ be two self maps on $X$. Let $\psi$ and $\beta$ be as defined in Theorem 1 with $\beta(r, s)=\beta(s, r)$. Then $T_{1}$ and $T_{2}$ have common unique fixed point, if for $x, y, a \in X$

$$
\psi\left(\left\|T_{1} x-T_{2} y, a\right\|\right) \leqslant \beta\left(\left\|x-T_{1} x, a\right\|,\left\|y-T_{2} y, a\right\|\right)-\max \left[\psi\left(\left\|x-T_{1} x, a\right\|\right), \psi\left(\left\|y-T_{2} y, a\right\|\right)\right]
$$

Proof. For a fixed point $x_{0} \in X$, we construct a sequence $\left\{x_{n}\right\}$ by

$$
x_{2 n+1}=T_{1}\left(x_{2 n}\right) \text { and } x_{2 n+2}=T_{2}\left(x_{2 n+1}\right), n=0,1,2, \ldots
$$

Now, it can be shown that $\left\{x_{n}\right\}$ is a Cauchy sequence in $X$, converging to some $z$ in $X$, which is the common fixed point for $T_{1}$ and $T_{2}$.

Corollary 1. Let X be a 2-Banach space and $T$ be a self map on X. Let $\psi, \beta$ be as defined in Theorem 3.1 satisfying

$$
\psi(\|T x-T y, a\|) \leqslant \frac{1}{c}[\beta(\|x-T y, a\|,\|y-T y, a\|)-\max (\psi(\|y-T x, a\|), \psi(\|x-T x, a\|))]
$$

where $c>2$ and $x, y, a \in X$. Then $T$ has unique fixed point on $X$.

Corollary 2. If $\psi$ satisfies then also similar result holds for

$$
\psi(\|T x-T y, a\|) \leqslant \beta(\|x-y, a\|,\|y-T y, a\|)-\max [\psi(\|x-y, a\|), \psi(\|x-T x, a\|)], \forall x, y, a \in X
$$

We now establish another fixed point theorem for $T$ using two other mappings $\gamma$ and $\phi$.

Theorem 3. Let $X$ be a 2-Banach space and $T$ be a self mapping on $X$. Let $\gamma:[0, \infty) \times[0, \infty) \times[0, \infty) \rightarrow[0, \infty)$ and $\phi:[0, \infty) \times[0, \infty) \rightarrow[0, \infty)$ be continuous mapping satisfying $\gamma(r, 0, r+t) \leqslant k r$ and $\phi(r, t) \geqslant k^{\prime} r$, where $k, k^{\prime} \in[0, \infty)$ such that $k-k^{\prime}<1$. Let

$$
\|T x-T y, a\| \leqslant \gamma[\|x-y, a\|,\|y-T x, a\|,\|x-T y, a\|]-\phi[\|x-T x, a\|,\|y-T y, a\|], \forall x, y, a \in X
$$

Then $T$ has a fixed point.

Proof. For any fixed $x_{0} \in X$, we construct a sequence $\left\{x_{n}\right\}$ by $x_{n+1}=T x_{n}$. We get,

$$
\begin{aligned}
\left\|x_{n}-x_{n+1}, a\right\| & \leqslant \gamma\left[\left\|x_{n-1}-x_{n}, a\right\|,\left\|x_{n}-x_{n}, a\right\|,\left\|x_{n-1}-x_{n+1}, a\right\|\right]-\phi\left[\left\|x_{n-1}-x_{n}, a\right\|,\left\|x_{n}-x_{n+1}, a\right\|\right] \\
& \leqslant \gamma\left[\left\|x_{n-1}-x_{n}, a\right\|, 0,\left\|x_{n-1}-x_{n}, a\right\|+\left\|x_{n}-x_{n+1}, a\right\|\right]-\phi\left[\left\|x_{n-1}-x_{n}, a\right\|,\left\|x_{n}-x_{n+1}, a\right\|\right] \\
& \leqslant\left(k-k^{\prime}\right)\left\|x_{n-1}-x_{n}, a\right\| \\
& \leqslant\left(k-k^{\prime}\right)^{2}\left\|x_{n-2}-x_{n-1}, a\right\| \leqslant \ldots \leqslant\left(k-k^{\prime}\right)^{n}\left\|x_{0}-x_{1}, a\right\| \rightarrow 0 \text { as } n \rightarrow \infty
\end{aligned}
$$

Hence $\left\{x_{n}\right\}$ is a Cauchy sequence in $X$ and so, it converges to some $z$,(say) in $X$.

$$
\begin{aligned}
\|z-T z, a\| & \leqslant\left\|z-x_{n+1}, a\right\|+\left\|x_{n+1}-T z, a\right\| \\
& \leqslant\left\|z-x_{n+1}, a\right\|+\gamma\left[\left\|x_{n}-z, a\right\|,\left\|z-T x_{n}, a\right\|,\left\|x_{n}-T z, a\right\|\right]-\phi\left[\left\|x_{n}-T x_{n}, a\right\|,\|z-T z, a\|\right] \\
& \leqslant 0+\gamma[0,0,0+\|z-T z, a\|]-\phi[0,\|z-T z, a\|][\text { taking } n \rightarrow \infty] .
\end{aligned}
$$

Therefore we write $\|z-T z, a\|=0$, for all $a \in X$. Since $a$ is arbitrary, taking $a=0$, we get, $z=T z$.

Example 2. Let $\gamma(r, s, t)=k_{1}(r+s+t)$ and $\phi(r, s)=k_{2}(r+s)$, where $k_{1}$ and $k_{2}$ are two constants $(>0)$. Now we can find out $k, k^{\prime} \in[0, \infty)$ with $k-k^{\prime}<1$ such that $\gamma(r, 0, r+t)=k_{1}(2 r+t) \leqslant k r$ and $\phi(r, t)=k_{2}(r+t) \geqslant k^{\prime} r$. Let $T$ and $X$ be as 
defined in Example 1. Now, for $x, y, a \in X$

$$
\|T x-T y, a\| \leqslant \gamma[\|x-y, a\|,\|y-T x, a\|,\|x-T y, a\|]-\phi[\|x-T x, a\|,\|y-T y, a\|]
$$

Hence by Theorem 3, $T$ has a fixed point on $X$.

Depending upon $k_{1}$ and $k_{2}$, the mapping $T$ is of different types. From the given condition,

$$
\begin{aligned}
\|T x-T y, a\| & \leqslant \gamma[\|x-y, a\|,\|y-x, a\|+\|x-T x, a\|,\|x-y, a\|+\|y-T y, a\|]-\phi[\|x-T x, a\|,\|y-T y, a\|] \\
& \leqslant k_{1}[3\|x-y, a\|+\|x-T x, a\|+\|y-T y, a\|]-k_{2}[\|x-T x, a\|+\|y-T y, a\|] .
\end{aligned}
$$

So, if $k_{1}=k_{2}$, then $\|T x-T y, a\| \leqslant 3 k_{1}\|x-y, a\|$ which is a contraction mapping for $k_{1}<\frac{1}{3}$ (and has a unique fixed point) and nonexpansive for $k_{1}=\frac{1}{3}$.

Next, we discuss common fixed point for four mappings in 2-Banach spaces.

\section{2-Norm for projective tensor product}

\subsection{Algebric tensor product}

[2]. Let $X, Y$ be normed spaces over $F$ with dual spaces $X^{*}$ and $Y^{*}$ respectively. Given $x \in X, y \in Y$, let $x \otimes y$ be the element of $B L\left(X^{*}, Y^{*} ; F\right)$ (which is the set of all bounded bilinear forms from $X^{*} \times Y^{*}$ to $F$ ), defined by

$$
x \otimes y(f, g)=f(x) g(y),\left(f \in X^{*}, g \in Y^{*}\right)
$$

The algebraic tensor product of $X$ and $Y, X \otimes Y$ is defined to be the linear span of $\{x \otimes y: x \in X, y \in Y\}$ in $B L\left(X^{*}, Y^{*} ; F\right)$.

\subsection{Projective tensor product}

[2]. Given normed spaces $X$ and $Y$, the projective tensor norm $\gamma$ on $X \otimes Y$ is defined by

$$
\|u\|_{\gamma}=\inf \left\{\sum_{i}\left\|x_{i}\right\|\left\|y_{i}\right\|: u=\sum_{i} x_{i} \otimes y_{i}\right\}
$$

where the infimum is taken over all (finite) representations of $u$. For the normed spaces $X$ and $Y$, in the projective tensor product $X \otimes_{\gamma} Y$, we take

$$
\|u, v\|=\|u\|\|v\|, u, v \in X \otimes_{\gamma} Y
$$

Following White [29], we can say that $X \otimes_{\gamma} Y$ is a 2-Banach space upto linear dependence (i.e., $X \otimes_{\gamma} Y$ satisfies all the conditions for being a 2-Banach space except $u$ and $v$ may be linearly dependent and yet $\|u, v\| \neq 0$ ).

Let $D_{X}, D_{Y}$ and $D_{X \otimes_{\gamma} Y}$ denote a closed and bounded subset of $X, Y$ and $X \otimes_{\gamma} Y$ respectively. Let $T_{1}$ and $T_{2}$ be two pairs of mappings where $T_{1}: D_{X \otimes_{\gamma} Y} \rightarrow D_{X}$ and $T_{2}: D_{X \otimes_{\gamma} Y} \rightarrow D_{Y}$ be such that for any $u, v \in D_{X \otimes_{\gamma} Y}$ and $a \otimes b \in D_{X \otimes_{\gamma} Y}$ with $\|a\| \geqslant 1$ and $\|b\| \geqslant 1$.

$(E)\left\|T_{1}(u)-T_{1}(v)\right\| \leqslant \frac{1}{K M_{2}}(k\|u-v, a \otimes b\|-\psi(k\|u-v, a \otimes b\|))$

$(F)\left\|T_{2}(u)-T_{2}(v)\right\| \leqslant \frac{1}{K M_{1}}\left(k^{\prime}\|u-v, a \otimes b\|-\psi\left(k^{\prime}\|u-v, a \otimes b\|\right)\right)$ 
where

(i) $\psi:[0, \infty) \rightarrow[0, \infty)$ is continuous and non-decreasing, $\psi(0)=0$

(ii) $\left\|T_{1} u\right\| \leqslant M_{1}$ and $\left\|T_{2} u\right\| \leqslant M_{2}, \forall u \in D_{X \otimes_{\gamma} Y}$.

Here, $D_{X \otimes_{\gamma} Y}$ is bounded by $K$ and $k, k^{\prime}$ are positive. From the mappings $T_{1}$ and $T_{2}$ we define a mapping $T: D_{X \otimes_{\gamma} Y} \rightarrow D_{X} \otimes_{\gamma} Y$ such that $T u=T_{1} u \otimes T_{2} u$.

Theorem 4. The mapping $T$ derived by the pair of mappings $\left(T_{1}, T_{2}\right)$ satisfying $(E)$ and $(F)$ has a unique fixed point in $D_{X \otimes \gamma}$ if $k+k^{\prime} \leqslant 1$.

Proof. For $u, v \in D_{X \otimes_{\gamma} Y}, a \in X$ and $b \in Y$ and $a \otimes b \in D_{X \otimes_{\gamma} Y}$ with $\|a\| \geqslant 1$ and $\|b\| \geqslant 1$

$$
\begin{aligned}
\|T u-T v, a \otimes b\| & =\left\|T_{1} u \otimes T_{2} u-T_{1} v \otimes T_{2} v, a \otimes b\right\| \\
& \leqslant\left\|\left(T_{1} u-T_{1} v\right) \otimes T_{2} u, a \otimes b\right\|+\left\|T_{1} v \otimes\left(T_{2} u-T_{2} v\right), a \otimes b\right\| \\
& =\left\|T_{1} u-T_{1} v\right\|\left\|T_{2} u\right\|\|a \otimes b\|+\left\|T_{1} v\right\|\left\|T_{2} u-T_{2} v\right\|\|a \otimes b\| \\
& \leqslant \frac{1}{K M_{2}}[k\|u-v, a \otimes b\|-\psi(k\|u-v, a \otimes b\|)] . K M_{2} \\
& +\frac{1}{K M_{1}}\left[k^{/}\|u-v, a \otimes b\|-\psi\left(k^{/}\|u-v, a \otimes b\|\right)\right] K M_{1} \\
& =\left(k+k^{\prime}\right)\|u-v, a \otimes b\|-\psi(k\|u-v, a \otimes b\|)-\psi\left(k^{\prime}\|u-v, a \otimes b\|\right) \\
& \leqslant\|u-v, a \otimes b\|-\left\{\psi(k\|u-v, a \otimes b\|)+\psi\left(k^{\prime}\|u-v, a \otimes b\|\right)\right\}
\end{aligned}
$$

Let $x_{0} \in D_{X \otimes_{\gamma} Y}$ be fixed. We take $x_{n+1}=T x_{n}$. Now,

$$
\begin{aligned}
\left\|x_{n+1}-x_{n}, a \otimes b\right\| & =\left\|T x_{n}-T x_{n-1}, a \otimes b\right\| \\
& \leqslant\left\|x_{n}-x_{n-1}, a \otimes b\right\|-\psi\left(k\left\|x_{n}-x_{n-1}, a \otimes b\right\|\right)-\psi\left(k^{/}\left\|x_{n}-x_{n-1}, a \otimes b\right\|\right) \\
& \leqslant\left\|x_{n}-x_{n-1}, a \otimes b\right\|
\end{aligned}
$$

Hence $\left\{\left\|x_{n+1}-x_{n}, a \otimes b\right\|\right\}$ is a monotonically decreasing sequence of non-negative real numbers and so, is convergent to some real, say $r$. Taking $n \rightarrow \infty$, we get

$$
r \leqslant r-\left\{\psi(k r)+\psi\left(k^{/} r\right)\right\},(\text { by continuity of } \psi) . \text { Then, } \psi(k r)+\psi\left(k^{/} r\right) \leqslant 0,
$$

this is possible only when $r=0$. So,

$$
\begin{aligned}
& \lim _{n \rightarrow \infty}\left\|x_{n+1}-x_{n}, a \otimes b\right\|=0 \\
& \Rightarrow \lim _{n \rightarrow \infty}\left\|x_{n+1}-x_{n}\right\|\|a \otimes b\|=0 \Rightarrow \lim _{n \rightarrow \infty}\left\|x_{n+1}-x_{n}\right\|=0 \\
& \Rightarrow \lim _{n \rightarrow \infty}\left\|x_{n+1}-x_{n}, u\right\|=0 \forall u \in D_{X \otimes_{\gamma} Y}
\end{aligned}
$$

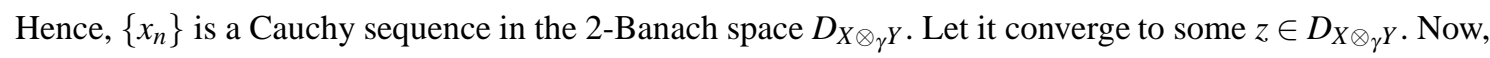

$$
\begin{aligned}
\|z-T z, u\| & \leqslant\left\|z-x_{n+1}, u\right\|+\left\|x_{n+1}-T z, u\right\|=\left\|z-x_{n+1}, u\right\|+\left\|T x_{n}-T z, u\right\| \\
& \leqslant\left\|z-x_{n+1}, u\right\|+\left\|x_{n}-z, u\right\|-\left[\psi\left(k\left\|x_{n}-z, u\right\|\right)+\psi\left(k^{/}\left\|x_{n}-z, u\right\|\right)\right] \\
& \rightarrow 0 \text { as } n \rightarrow \infty
\end{aligned}
$$


Hence, $\|z-T z, u\|=0 \Rightarrow z=T z$. To show the uniqueness. Let $z_{1}$ and $z_{2}$ be two distinct fixed points for $T$ in $D_{X \otimes_{\gamma} Y}$. Now,

$$
\begin{aligned}
\left\|z_{1}-z_{2}, u\right\|=\left\|T z_{1}-T z_{2}, u\right\| & \leqslant\left\|z_{1}-z_{2}, u\right\|-\left[\psi\left(k\left\|z_{1}-z_{2}, u\right\|\right)+\psi\left(k^{\prime}\left\|z_{1}-z_{2}, u\right\|\right)\right] \\
& \Rightarrow \psi\left(k\left\|z_{1}-z_{2}, u\right\|\right)+\psi\left(k^{\prime}\left\|z_{1}-z_{2}, u\right\|\right) \leqslant 0
\end{aligned}
$$

which is contradiction. So, $z_{1}=z_{2}$. Thus, $T$ has a unique fixed point in the closed and bounded subset $D_{X \otimes_{\gamma} Y}$ of $X \otimes_{\gamma} Y$.

Example 3. Let $D_{l^{1} \otimes_{\gamma} \mathbb{K}}$ (with the same 2-norm as defined above in the tensor product space), $D_{l^{1}}$ and $D_{\mathbb{K}}$ denote a closed and bounded subset of $l^{1} \otimes_{\gamma} \mathbb{K}, l^{1}$ and $\mathbb{K}$, bounded by $K, \sqrt{K}$ and $\sqrt{K}$ respectively $(K>0)$.

We define $T_{1}: D_{l^{1} \otimes \mathbb{Y}_{\mathbb{K}}} \rightarrow D_{l^{1}}$ by

$$
T_{1}\left(\sum_{i} a_{i} \otimes x_{i}\right)=\frac{1}{2 K^{3}} \sum_{i}\left\{a_{i_{n}} x_{i}\right\}, \text { where } a_{i}=\left\{a_{i_{n}}\right\}_{n}
$$

and $T_{2}: D_{l^{1} \otimes \mathbb{\gamma}^{K}} \rightarrow D_{\mathbb{K}} \quad$ by $\quad T_{2}\left(\sum_{i} a_{i} \otimes x_{i}\right)=\frac{1}{4} \sum_{i}\left\|a_{i}\right\| \cdot\left|x_{i}\right|$. For arbitrary $b_{k}=\left\{b_{k_{n}}\right\} \in D_{l^{1}}, b \in D_{\mathbb{K}}$ with $\left\|b_{k}\right\| \geqslant 1$ and $|b| \geqslant 1$,

$$
\begin{aligned}
\left\|T_{1}\left(\sum_{i} a_{i} \otimes x_{i}\right)\right\| & =\left\|\frac{1}{2 K^{3}} \sum_{i}\left\{a_{i_{n}} x_{i}\right\}\right\| \leqslant \frac{1}{2 K^{3}} \sum_{i}\left\|\left\{a_{i_{n}} x_{i}\right\}\right\|\left\|b_{k}\right\||b| \\
& \leqslant \frac{1}{2 K^{3}}\left\|\sum_{i} a_{i} \otimes x_{i}\right\|\left\|b_{k} \otimes b\right\|\left[l^{1} \otimes \gamma X=l^{1}(X)(\text { refer to [26])] }\right. \\
& \leqslant \frac{1}{2 K^{3}} K^{2}=\frac{1}{2 K}\left(=M_{1}\right),
\end{aligned}
$$

and

$$
\left\|T_{2}\left(\sum_{i} a_{i} \otimes x_{i}\right)\right\| \leqslant \frac{1}{4}\left\|\sum_{i} a_{i} \otimes x_{i}\right\|\left\|b_{k} \otimes b\right\| \leqslant \frac{K^{2}}{4}\left(=M_{2}\right)
$$

For $u=\sum_{i} a_{i} \otimes x_{i}$ and $v=\sum_{i} d_{i} \otimes y_{i}$ in $D_{l^{1} \otimes} \mathbb{K}$, we have,

$$
\begin{aligned}
\left\|T_{1} u-T_{1} v\right\| & =\left\|\frac{1}{2 K^{3}} \sum_{i}\left\{a_{i_{n}} x_{i}\right\}-\frac{1}{2 K^{3}} \sum_{i}\left\{d_{i_{n}} y_{i}\right\}\right\| \\
& \left.=\frac{\frac{1}{4}\left\|\sum_{i} a_{i} \otimes x_{i}-\sum_{i} d_{i} \otimes y_{i}\right\|}{\frac{K^{3}}{2}} \leqslant \frac{\frac{1}{4}\|u-v\|\left\|b_{k} \otimes b\right\|}{\frac{K^{3}}{2}}\right] \\
& \leqslant 2\left[\frac{\frac{1}{2}\left\|u-v, b_{k} \otimes b\right\|-\frac{1}{2}\left[\frac{1}{2}\left\|u-v, b_{k} \otimes b\right\|\right]}{K \frac{K^{2}}{2}}\right] \\
& =\frac{1}{K M_{2}}\left[\frac{1}{2}\left\|u-v, b_{k} \otimes b\right\|-\psi\left(\frac{1}{2}\left\|u-v, b_{k} \otimes b\right\|\right)\right] ; \text { where } \psi(t)=\frac{t}{2}, k=\frac{1}{2},
\end{aligned}
$$

and

$$
\left\|T_{2} u-T_{2} v\right\|=\left\|\frac{1}{4} \sum_{i}\right\| a_{i}\left\|\cdot\left|x_{i}\right|-\frac{1}{4} \sum_{i}\right\| d_{i}\left\|\cdot\left|y_{i}\right|\right\| \leqslant \frac{1}{4}\left|\sum_{i}\left\|a_{i}\right\| \cdot\right| x_{i}\left|-\sum_{i}\left\|d_{i}\right\| \cdot\right| y_{i}||\left\|b_{k}\right\||| b \mid .
$$


Taking the projective tensor norm,

$$
\begin{aligned}
\left\|T_{2} u-T_{2} v\right\| & \leqslant \frac{1}{4}|\|u\|-\|v\||\left\|b_{k} \otimes b\right\| \leqslant \frac{1}{4}\|u-v\|\left\|b_{k} \otimes b\right\|=\frac{1}{4}\left\|u-v, b_{k} \otimes b\right\| \\
& \leqslant \frac{\frac{1}{2}\left\|u-v, b_{k} \otimes b\right\|-\frac{1}{2}\left[\frac{1}{2}\left\|u-v, b_{k} \otimes b\right\|\right]}{K \frac{1}{2 K}} \\
& \leqslant \frac{\frac{1}{2}\left\|u-v, b_{k} \otimes b\right\|-\psi\left(\frac{1}{2}\left\|u-v, b_{k} \otimes b\right\|\right)}{K M_{1}} ; \text { where } \psi(t)=\frac{t}{2}, k^{\prime}=\frac{1}{2}
\end{aligned}
$$

Therefore, $\left(T_{1}, T_{2}\right)$ satisfies the conditions $(a)$ and $(b)$. Also, $k+k^{\prime}=\frac{1}{2}+\frac{1}{2}=1$. So, the mapping $T: D_{l^{1} \otimes_{\gamma} \mathbb{K}} \rightarrow D_{l^{1} \otimes \gamma \mathbb{K}}$ has a unique fixed point in $D_{l^{1} \otimes \gamma \mathbb{K}}$.

Let $T_{1}, S_{1}, P_{1}, T_{2}, S_{2}$ and $P_{2}$ be some mappings where $T_{1}, S_{1}, P_{1}: D_{X \otimes_{\gamma} Y} \rightarrow D_{X}$ and $T_{2}, S_{2}, P_{2}: D_{X \otimes_{\gamma} Y} \rightarrow D_{Y}$ be two mappings such that for any $u, v \in D_{X \otimes_{\gamma} Y}$ and $a \otimes b \in X \otimes_{\gamma} Y$,

$(G)\left\|T_{1}(u)-S_{1}(v)\right\| \leqslant \frac{1}{M M_{2}}(k(\|P u-T v, a \otimes b\|+\|P u-S v, a \otimes b\|)-\psi(k\|P u-T v, a \otimes b\|, k\|P u-S v, a \otimes b\|))$

$(H)\left\|T_{2}(u)-S_{2}(v)\right\| \leqslant \frac{1}{M M_{1}}\left(k^{/}(\|P u-T v, a \otimes b\|+\|P u-S v, a \otimes b\|)-\psi\left(k^{/}\|P u-T v, a \otimes b\|, k^{/}\|P u-S v, a \otimes b\|\right)\right)$

where

(i) $\psi:[0, \infty) \rightarrow[0, \infty)$ is continuous and non-decreasing, $\psi(0)=0$

(ii) $\max \left[\left\|T_{1} u\right\|,\left\|S_{1} v\right\|\right] \leqslant M_{1}$ and $\max \left[\left\|T_{2} u\right\|,\left\|S_{2} v\right\|\right] \leqslant M_{2}, \forall u, v \in D_{X \otimes_{\gamma} Y}, a \in X$ and $b \in Y$. Here, $D_{X \otimes_{\gamma} Y}$ is bounded by $M$ and $k, k^{\prime}$ are positive.

From the mappings $T_{1}, S_{1}, P_{1}, T_{2}, S_{2}$ and $P_{2}$ we define some mappings $T: D_{X \otimes_{\gamma} Y} \rightarrow D_{X \otimes_{\gamma} Y}$ such that $T u=T_{1} u \otimes T_{2} u$; $S: D_{X \otimes_{\gamma} Y} \rightarrow D_{X \otimes_{\gamma} Y}$ such that $S u=S_{1} u \otimes S_{2} u$ and $P: D_{X \otimes_{\gamma} Y} \rightarrow D_{X \otimes_{\gamma} Y}$ such that $P u=P_{1} u \otimes P_{2} u$.

Theorem 5. Let T,S and $P$ be self mappings as defined above such that

(i) $\{T, P\}$ and $\{S, P\}$ are wealy compatible

(ii) $T\left(X \otimes_{\gamma} Y\right) \subseteq P\left(X \otimes_{\gamma} Y\right)$ and $S\left(X \otimes_{\gamma} Y\right) \subseteq P\left(X \otimes_{\gamma} Y\right)$

(iii) satisfy $(G)$ and $(H)$, then $T, S$ and $P$ have a common unique fixed point on $D_{X \otimes_{\gamma} Y}$ if $k+k / \leqslant \frac{1}{4}$

Proof. Let $x_{0} \in D_{X \otimes_{\gamma} Y}$ be fixed. We define

$$
y_{n}=T x_{n}=P x_{n+1}, y_{n+1}=S x_{n+1}=P x_{n+2}
$$

Now, for any $a \otimes b \in D_{X \otimes_{\gamma} Y}$,

$$
\begin{aligned}
\|T u-S v, a \otimes b\| & \leqslant\left\|T_{1} u-S_{1} v\right\|\left\|T_{2} u\right\|\|a \otimes b\|+\left\|S_{1} v\right\|\left\|T_{2} u-S_{2} v\right\|\|a \otimes b\| \\
& \leqslant \frac{1}{4}(\|P u-T v, a \otimes b\|+\|P u-S v, a \otimes b\|)-\psi\left(k^{/}\|P u-T v, a \otimes b\|, k^{/}\|P u-S v, a \otimes b\|\right) .
\end{aligned}
$$




$$
\begin{aligned}
\left\|y_{n}-y_{n+1}, a \otimes b\right\| & =\left\|T x_{n}-S x_{n+1}, a \otimes b\right\| \\
& \leqslant \frac{1}{4}\left(\left\|P x_{n}-T x_{n+1}, a \otimes b\right\|+\left\|P x_{n}-S x_{n+1}, a \otimes b\right\|\right) \\
& -\psi\left(k\left\|P x_{n}-T x_{n+1}, a \otimes b\right\|, k\left\|P x_{n}-S x_{n+1}, a \otimes b\right\|\right) \\
& -\psi\left(k^{/}\left\|P x_{n}-T x_{n+1}, a \otimes b\right\|, k^{/}\left\|P x_{n}-S x_{n+1}, a \otimes b\right\|\right) \\
& =\frac{1}{4}\left(\left\|y_{n-1}-y_{n+1}, a \otimes b\right\|+\left\|y_{n-1}-y_{n+1}, a \otimes b\right\|\right) \\
& -\psi\left(k\left\|y_{n-1}-y_{n+1}, a \otimes b\right\|, k\left\|y_{n-1}-y_{n+1}, a \otimes b\right\|\right) \\
& -\psi\left(k^{/}\left\|y_{n-1}-y_{n+1}, a \otimes b\right\|, k^{\prime}\left\|y_{n-1}-y_{n+1}, a \otimes b\right\|\right) \\
& \leqslant \frac{1}{2}\left(\left\|y_{n-1}-y_{n}, a \otimes b\right\|+\left\|y_{n}-y_{n+1}, a \otimes b\right\|\right) \\
& -\psi\left(k\left\|y_{n-1}-y_{n+1}, a \otimes b\right\|, k\left\|y_{n-1}-y_{n+1}, a \otimes b\right\|\right) \\
& -\psi\left(k^{/}\left\|y_{n-1}-y_{n+1}, a \otimes b\right\|, k^{\prime}\left\|y_{n-1}-y_{n+1}, a \otimes b\right\|\right) \\
& =\left\|y_{n-1}-y_{n}, a \otimes b\right\|-2 \psi\left(k\left\|y_{n-1}-y_{n+1}, a \otimes b\right\|, k\left\|y_{n-1}-y_{n+1}, a \otimes b\right\|\right) \\
& -2 \psi\left(k^{\prime}\left\|y_{n-1}-y_{n+1}, a \otimes b\right\|, k^{/}\left\|y_{n-1}-y_{n+1}, a \otimes b\right\|\right) \\
& \leqslant\left\|y_{n-1}-y_{n}, a \otimes b\right\| .
\end{aligned}
$$

Hence $\left\{\left\|y_{n+1}-y_{n}, a \otimes b\right\|\right\}$ is a monotonically decreasing sequence of non-negative real numbers and so, is convergent to some real, say $r$. If $r \neq 0$, then

$$
\begin{aligned}
\left\|y_{n}-y_{n+1}, a \otimes b\right\| & =\left\|T x_{n}-S x_{n+1}, a \otimes b\right\| \\
& \leqslant \frac{1}{4}\left(\left\|y_{n-1}-y_{n+1}, a \otimes b\right\|+\left\|y_{n-1}-y_{n+1}, a \otimes b\right\|\right) \\
& -\psi\left(k\left\|y_{n-1}-y_{n+1}, a \otimes b\right\|, k\left\|y_{n-1}-y_{n+1}, a \otimes b\right\|\right) \\
& -\psi\left(k^{/}\left\|y_{n-1}-y_{n+1}, a \otimes b\right\|, k^{\prime}\left\|y_{n-1}-y_{n+1}, a \otimes b\right\|\right) \\
& \leqslant \frac{1}{2}\left(\left\|y_{n-1}-y_{n+1}, a \otimes b\right\|\right) \leqslant \frac{1}{2}\left(\left\|y_{n-1}-y_{n}, a \otimes b\right\|+\left\|y_{n}-y_{n+1}, a \otimes b\right\|\right)
\end{aligned}
$$

Taking $n \rightarrow \infty$, we get $\left\|y_{n-1}-y_{n+1}, a \otimes b\right\| \rightarrow 2 r$ and

$r \leqslant r-2\left\{\psi(2 k r, 2 k r)+\psi\left(2 k^{/} r, 2 k^{/} r\right)\right\},($ by continuity of $\psi)$, therefore $2\left\{\psi(2 k r, 2 k r)+\psi\left(2 k^{/} r, 2 k^{/} r\right)\right\} \leqslant 0$

this is possible only when $r=0$. So,

$$
\lim _{n \rightarrow \infty}\left\|y_{n+1}-y_{n}, a \otimes b\right\|=0
$$

Now, proceeding as in Theorem 4.3 we have $\lim _{n \rightarrow \infty}\left\|y_{n+1}-y_{n}, q\right\|=0 \forall q \in D_{X \otimes_{\gamma} Y}$ and $\left\{y_{n}\right\}$ is a Cauchy sequence in $D_{X \otimes_{\gamma} Y}$. Let it converge to some $z \in D_{X \otimes_{\gamma} Y}$ i.e.,

$$
\begin{aligned}
\lim _{n \rightarrow \infty} y_{n}=z \Rightarrow & \lim _{n \rightarrow \infty} T x_{n}=\lim _{n \rightarrow \infty} P x_{n+1}=z \text { and } \\
\lim _{n \rightarrow \infty} S x_{n+1} & =\lim _{n \rightarrow \infty} P x_{n+2}=z .
\end{aligned}
$$


Since $S(X) \subseteq P(X)$ and $T(X) \subseteq P(X)$, so there exists a point $u \in D_{X \otimes_{\gamma} Y}$ such that $z=P u$. Now,

$$
\begin{aligned}
\|T u-z, q\| & \leqslant\left\|T u-S x_{n+1}, q\right\|+\left\|S x_{n+1}-z, q\right\| \\
& \leqslant \frac{1}{4}\left(\left\|P u-T x_{n+1}, q\right\|+\left\|P u-S x_{n+1}, q\right\|\right) \\
& -\psi\left(k\left\|P u-T x_{n+1}, q\right\|, k\left\|P u-S x_{n+1}, q\right\|\right) \\
& -\psi\left(k^{\prime}\left\|P u-T x_{n+1}, q\right\|, k^{/}\left\|P u-S x_{n+1}, q\right\|\right)+\left\|S x_{n+1}-z, q\right\| .
\end{aligned}
$$

Taking $n \rightarrow \infty$,

$$
\|T u-z, q\| \leqslant 0 \Rightarrow\|T u-z, q\|=0
$$

Therefore $T u=z$. So, $P u=T u=z$, i.e., $u$ is a coincidence point of $P$ and $T$. Since the pair of mappings are weakly compatiable, so,

$$
P T u=T P u \Rightarrow P z=T z
$$

Again for $z=P u$ we have,

$$
\begin{aligned}
\|z-S u, q\| & =\|T u-S u, q\| \\
& \leqslant \frac{1}{4}(\|P u-T u, q\|+\|P u-S u, q\|) \\
& -\psi(k\|P u-T u, q\|, k\|P u-S u, q\|) \\
& -\psi\left(k^{/}\|P u-T u, q\|, k^{\prime}\|P u-S u, q\|\right)=0 .
\end{aligned}
$$

Thus, $\|z-S u, q\|=0$. So, $S u=z$. Thus $P u=S u=z$, i.e., $w$ is a coincidence point of $P$ and $S$. Since the pair of mappings are weakly compatiable, so,

$$
P S u=S P u \Rightarrow P z=S z
$$

Now, we show that $z$ is a fixed point of $T$

$$
\begin{aligned}
\|T z-z, q\| & =\|T z-S u, q\| \\
& \leqslant \frac{1}{4}(\|P z-T u, q\|+\|P z-S u, q\|) \\
& -\psi(k\|P z-T u, q\|, k\|P z-S u, q\|) \\
& -\psi\left(k^{/}\|P z-T u, q\|, k^{/}\|P z-S u, q\|\right) \\
& =\frac{1}{2}\|T z-z, q\|
\end{aligned}
$$

possible only for $\|T z-z, q\|=0 \Rightarrow T z=z$ therefore $T z=P z=z$. Now, we show that $z$ is a fixed point of $S$

$$
\begin{aligned}
\|z-S z, q\|= & \|T z-S z, q\| \\
\leqslant \frac{1}{4}( & \|P z-T z, q\|+\|P z-S z, q\|) \\
& \quad-\psi(k\|P z-T z, q\|, k\|P z-S z, q\|) \\
& \quad-\psi\left(k^{/}\|P z-T z, q\|, k^{/}\|P z-S z, q\|\right)=0
\end{aligned}
$$


possible only for $S z=z$ therefore $S z=P z=z$. Hence, $T z=P z=z=S z$. Uniqueness can be shown in a similar manner. Thus $z$ is a common unique fixed point for the mappings $T, S$ and $P$.

\section{Conclusion}

Thus, in this paper, we have derived different fixed point theorems in 2-Banach spaces and also in the tensor product of normed spaces as 2-Banach spaces.

In the paper of Misiak [17], in 1989, the idea of $n$-normed spaces can be found. Some recent results and related works in $n$-normed spaces can be found in [13], [16]. Let $n \in \mathbb{N}$ and $X$ be a real vector space of dimension $d \geqslant n$. A real-valued function $\|., \ldots,$.$\| on X^{n}$ satisfying the following four properties,

(1) $\left\|x_{1}, \ldots, x_{n}\right\|=0$ if and only if $x_{1}, \ldots, x_{n}$ are linearly dependent;

(2) $\left\|x_{1}, \ldots, x_{n}\right\|$ is invariant under permutation;

(3) $\left\|x_{1}, \ldots, x_{n-1}, \alpha x_{n}\right\|=|\alpha|\left\|x_{1}, \ldots, x_{n-1}, x_{n}\right\|$ for any $\alpha \in \mathbb{R}$;

(4) $\left\|x_{1}, \ldots, x_{n-1}, y+z\right\| \leqslant\left\|x_{1}, \ldots, x_{n-1}, y\right\|+\left\|x_{1}, \ldots, x_{n-1}, z\right\|$, is called an $n$-norm on $X$ and the pair $(X,\|., \ldots,\|$.$) is called an n$-normed space. Considering the study of fixed points, the following problem can be raised.

Can we make analogous study concerning fixed points for a mapping $T$ in the $n$-normed spaces and their tensor product?

\section{Competing interests}

The authors declare that they have no competing interests.

\section{Authors' contributions}

All authors have contributed to all parts of the article. All authors read and approved the final manuscript.

\section{References}

[1] M.Ackgoz, A review on 2-normed structures, Int. Journal of Math. Analysis, 1(2007), no.4, 187 - 191. MR2340939 (2008e:46012) Zbl 1132.46304

[2] F. F. Bonsal and J. Duncan, Complete Normed algebras, Springer-Verlag, Berlin Heidelberg New York, 1973

[3] Y.J.Cho, M.S.Khan and S.L.Sing, Common fixed points of weakly commuting mappings, Univ.u. Novom Sadu, Zb.Rad. Period.Mat.Fak.Ser.Mat, 181(1988)129-142. MR1034710

[4] D. Das, N. Goswami, Fixed Points of Different Contractive Type Mappings on Tensor Product Spaces, IJIRSET, Vol.3, July 2014, No.7.

[5] D. Das, N. Goswami,Fixed Points of Mapping Satisfying a Weakly Contractive Type Condition, Journal of Math. Res. with Appl., Vol 36(2016), No. 1 pp. 70-78

[6] Deepmala and H. K. Pathak, A study on some problems on existence of solutions for nonlinear functional-integral equations, Acta Mathematica Scientia, 33 B(5) (2013), 1305-1313.

[7] Deepmala, A Study on Fixed Point Theorems for Nonlinear Contractions and its Applications, Ph.D. Thesis (2014), Pt. Ravishankar Shukla University, Raipur 492 010, Chhatisgarh, India. 
[8] Deepmala, L.N. Mishra, Differential operators over modules and rings as a path to the generalized differential geometry, FACTA UNIVERSITATIS (NI Š) Ser. Math. Inform. Vol. 30, No. 5 (2015), pp. 753-764.

[9] S. Elumalai, R.Vijayaragavan, Characterizations of best approximations in linear 2-normed spaces, General Mathematics Vol. 17 , No. 3 (2009), 141-160

[10] S. Gähler, Lineare 2-normierte Riume, Math. Nachr. 28 (1965), 1-43.

[11] K. Iseki, Fixed point theorems in 2-metric space, Math.Seminar.Notes, Kobe Univ.,3(1975), 133 - 136. MR0405395

[12] M.S. Khan and M.D. Khan, Involutions with Fixed Points in 2-Banach Spaces, Internat. J. Math. and Math. Sci. VOL. 16 NO. 3 (1993) 429-434.

[13] S. S. Kim and Y.J. Cho, Strict Convexity in linear n-normed spaces, Demonstratio Math. 29(1996), no. 4, 739-744

[14] İ. Kisi, S. Büyükkütük, Deepmala, G. Ozturk, AW(k)-type Curves According to Parallel Transport Frame in Euclidean Space $\mathbb{E}^{4}$, FACTA UNIVERSITATIS (NIÅ) Ser. Math. Inform. Vol. 31, No. 4 (2016).

[15] Z. Liu, X. Zhang, J. Sheok Ume and S. Min Kang, Common fixed point theorems for four mappings satisfying $\psi$-weakly contractive conditions, Fixed Point Theory and Applications (2015), 1-22

[16] R. Malčeski, Strong n-convex n-normed spaces, Mat. Bilten 21(1997), 81-102 MR 99m:46059

[17] A. Misiak, n-inner product spaces, Math. Nachr. 140 (1989), 299-319. MR 91a:46021

[18] L.N. Mishra, S.K. Tiwari, V.N. Mishra; Fixed point theorems for generalized weakly S-contractive mappings in partial metric spaces, Journal of Applied Analysis and Computation, Volume 5, Number 4, 2015, pp. 600-612. doi:10.11948/2015047

[19] L.N. Mishra, On existence and behavior of solutions to some nonlinear integral equations with Applications, Ph.D. Thesis (2016), National Institute of Technology, Silchar 788 010, Assam, India.

[20] L.N. Mishra, M. Sen, R.N. Mohapatra, On existence theorems for some generalized nonlinear functional-integral equations with applications, Filomat, accepted on March 21, 2016, in press.

[21] L. N. Mishra, R. P. Agarwal, M. Sen, Solvability and asymptotic behavior for some nonlinear quadratic integral equation involving Erdélyi-Kober fractional integrals on the unbounded interval, Progress in Fractional Differentiation and Applications Vol. 2, No. 3 (2016), 153-168.

[22] L.N. Mishra, H.M. Srivastava, M. Sen, On existence results for some nonlinear functional-integral equations in Banach algebra with applications, Int. J. Anal. Appl., Vol. 11, No. 1, (2016), 1-10.

[23] L.N. Mishra, M. Sen, On the concept of existence and local attractivity of solutions for some quadratic Volterra integral equation of fractional order, Applied Mathematics and Computation Vol. 285, (2016), 174-183. DOI: 10.1016/j.amc.2016.03.002

[24] L.N. Mishra, S.K. Tiwari, V.N. Mishra, I.A. Khan; Unique Fixed Point Theorems for Generalized Contractive Mappings in Partial Metric Spaces, Journal of Function Spaces, Volume 2015 (2015), Article ID 960827, 8 pages.

[25] H.K. Pathak and Deepmala, Common fixed point theorems for PD-operator pairs under Relaxed conditions with applications, Journal of Computational and Applied Mathematics, 239 (2013), 103-113.

[26] A. Raymond Ryan, Introduction to Tensor Product of Banach Spaces, London,Springer -Verlag,2002.

[27] B.E. Rhoades, Contractive type mappings on a 2-metric space, Math.Nachr.,91(1979), 151 - 155. MR0563606

[28] M. Saha, D. Dey, A. Ganguly and L. Debnath, Fixed Point Theorems for a Class of Weakly C-Contractive Mappings in a Setting of 2-Banach Space, Journal of Mathematics, Volume 2013, Article ID 434205, 7 pages

[29] A. White, 2-Banach spaces, Math.Nachr., 42(1969), 43 - 60. MR0257716 (41 2365) Zbl 0185.20003 